\title{
A Aplicação da Neuropsicologia na Pesquisa EXPERIMENTAL: O EXEMPLO DA INTOXICAÇÃO POR VAPOR DE MERCÚRIO
}

\author{
Anita Taub ${ }^{1}$, Elaine Cristina Zachi ${ }^{2}$, Rosani Aparecida Antunes \\ Teixeira ${ }^{2}$, Marcília da Araújo Medrado Faria ${ }^{3}$ e Dora Fix Ventura ${ }^{4}$ \\ Universidade de São Paulo - USP
}

O desenvolvimento da neuropsicologia acompanhou a evolução do estudo do cérebro desde os registros mais antigos. A ciência neuropsicológica faz. uso de medidas quantitativas de desempenho e tecnologias para produção de neuroimagem que, em conjunto, permitem a observação das relações cérebro-comportamento. No âmbito experimental, a neuropsicologia tem revelado novos conhecimentos na investigação de alterações cognitivas, psicomotoras ou comportamentais associadas a patologias congênitas ou adquiridas. No presente artigo são abordados aspectos históricos da neuropsicologia experimental e apresentados os principais resultados de um estudo referente às alterações neuropsicológicas de pacientes com histórico de intoxicação por vapor de mercúrio, presentes anos após o período de exposição ao metal.

Descritores: Neuropsicologia. Avaliação neuropsicológica. Intoxicação por mercúrio.

Neuropsicologia é o ramo da neurociência que estuda a relação entre o
comportamento e o funcionamento cerebral em condições normais ou

1 Pesquisadora do PROTOC - Projeto Transtorno Obsessivo-Compulsivo do Instituto de Psiquiatria da Faculdade de Medicina - USP. Endereço eletrônico: anita_taub@uol.com.br

2 Pós-Graduandas do Programa de Neurociências e Comportamento do Instituto de Psicologia - USP.

3 Docente do Departamento de Medicina Legal, Ética Médica e Medicina Social e do Trabalho da Faculdade de Medicina - USP.

4 Docente do Departamento de Psicologia Experimental do Instituto de Psicologia - USP. 
patológicas, decorrentes de fatores congênitos, lesões adquiridas ou disfunções de natureza elétrica ou química (Gil, 2002). Sua história nos remete ao próprio estudo do cérebro, cujos primeiros registros foram encontrados na Antigüidade.

Pitágoras trouxe as observações mais remotas do cérebro como o órgão responsável pelo pensamento e pelas sensações, com áreas específicas relativas às diferentes modalidades sensoriais como a visão, a audição, etc (Feinberg \& Farah, 1997). A partir de então, o estudo do cérebro nunca deixou de intrigar cientistas das mais variadas áreas de especialidade, principalmente através de observações anatômicas, na tentativa de localizar as funções cerebrais. No século XIX, o anatomista Franz Joseph Gall trouxe importantes contribuições ao estudo do cérebro a partir da compreensão de que as diferentes faculdades mentais humanas estavam localizadas em áreas cerebrais distintas e que o cérebro não funcionava como um órgão unitário, mas sim, subdividido em várias unidades, cada uma delas correspondendo a uma faculdade mental específica ou traço de comportamento. Apoiado na hipótese de localização estreita, Gall desenvolveu a chamada frenologia (phrenos $=$ mente, $\log o s=$ estudo), que propôs um método para prever traços de personalidade e de desenvolvimento das faculdades mentais e morais através do formato do crânio. Embora este modelo tenha sido muito popular durante o século XIX, ele passou a ser amplamente questionado e foi abandonado devido à ausência de bases científicas ou observações clínicas.

Na segunda metade do século XIX, com tendência ainda localizacionista, porém caracterizada por pesquisas científicas e seguindo o modelo lesional, novos estudos promoveram avanços na área da neurologia comportamental. Em 1861, Paul Broca, (1824-1880) em sua investigação científica dos distúrbios dos processos mentais, foi pioneiro na identificação da área cerebral envolvida na fala expressiva com a descrição do caso de um paciente com dificuldade na expressão da fala acompanhada da destruição do terço posterior do giro frontal inferior no hemisfério esquerdo, no qual a compreensão da linguagem estava preservada. Posteriormente, o psiquiatra Carl Wernicke (1848-1904) constatou que o giro temporal superior esquerdo se associa à capacidade de compreender a fala audível, descrevendo o caso de um paciente com uma lesão nesta área com comprometimento significativo da habilidade de compreender a linguagem falada, porém, com a linguagem expressiva preservada (Luria, 1981). 
A Aplicação da Neuropsicologia na Pesquisa Experimental: o Exemplo...

A partir do século XX, a tendência estritamente localizacionista da atividade mental foi questionada e a noção de "sistema funcional" foi difundida pelo neurologista Alexander Romanovich Luria (1901-1978), considerado o fundador da vertente soviética da Neuropsicologia e um marco para este corpo de conhecimento. Esta nova teoria acerca do funcionamento cerebral desenvolveu-se através do estudo do impacto comportamental de lesões em diferentes regiões do cérebro, permitindo correlacionar disfunções em diferentes regiões a alterações do comportamento. Com base em suas observações, Luria elaborou o conceito de sistema funcional, segundo o qual as funções mentais são organizadas em "sistemas funcionais complexos", formados por estruturas nervosas com papéis específicos que atuam em concerto para a execução de determinada habilidade mental. De acordo com este modelo, todo o sistema funcional pode ser perturbado em casos de uma lesão em área cerebral específica ou múltiplas lesões em regiões diferentes (Luria, 1981).

Seguindo o modelo de estudo de pacientes com lesões cerebrais, Brenda Milner trouxe, na década de 60, novas perspectivas para a compreensão da memória e sua dissociação entre curta e longa duração, através da descrição do paciente H. M., que foi submetido a remoção bilateral de parte do córtex temporal medial, amígdala e parte do hipocampo visando extinguir crises epilépticas. Como sequiela, a paciente não era capaz de formar novos registros mnemônicos, não se lembrava de fatos e eventos ocorridos imediatamente antes da cirurgia, mas podia recuperar memórias anteriores (Magila \& Xavier, 2000). Em 1975, Milner avaliou pacientes epilépticos submetidos à cirurgia dos lobos temporal e frontal através de baterias neuropsicológicas pré e pós-procedimento cirúrgico e descreveu as alterações de comportamento após ablação bilateral do lobo temporal, o que permitiu o desenvolvimento de instrumentos para a avaliação de memória e funções executivas.

A neuropsicologia experimental resultou de avanços provenientes de estudos em pacientes únicos com distúrbios particulares (Feinberg \& Farah, 1997), importantes pela observação e descrição de alterações cerebrais e respectivas manifestações comportamentais. A verificação de que as funções mentais complexas se relacionam ao funcionamento de áreas cerebrais atuantes em concerto possibilitou a associação entre cérebro e comportamento, o que constitui a base da neuropsicologia. O termo "Neuropsicologia" foi apresentado de maneira sistemática a partir de 1913 (Lezak, 1995). 
A aplicação dos conhecimentos da Neuropsicologia na esfera da investigação científica apresenta características distintas de sua aplicação no contexto clínico, cujos objetivos principais são o diagnóstico e a reabilitação de pacientes com disfunções cognitivas de desenvolvimento ou adquiridas. Com o avanço das novas tecnologias e de recursos que possibilitam a investigação do cérebro em funcionamento, como a Ressonância magnética funcional (RMf), a Tomografia por emissão de fóton único (SPECT) e Tomografia por emissão de pósitrons (PET), desenvolveram-se instrumentos de investigação neuropsicológica de aplicação à pesquisa experimental, com medidas quantitativas de desempenho, possibilitando uma melhor compreensão das relações cérebro-comportamento. De acordo com o desenho de cada estudo, são utilizados testes padronizados ou tarefas (paradigmas) desenvolvidas para testar uma hipótese específica, ou ainda, observar de que modo o cérebro responde a esta tarefa, caso da RMf. Nos últimos anos, estudos brasileiros têm utilizado a RMf na estimação acurada da lateralização da memória e da linguagem (Branco \& da Costa, 2006; Meneses et al., 2004), no estudo da modulação do comportamento através de estímulos emocionais (Volchan et al., 2003), verificar o correlato de ativação cerebral durante experiências emocionais (Moll et al., 2002), entre outras finalidades.

O uso de instrumentos padronizados nos permite comparar o funcionamento cognitivo de indivíduos saudáveis com indivíduos em condições patológicas específicas através de seu desempenho nos testes neuropsicológicos. Neste tipo de estudo, são utilizados protocolos de avaliação fechados com instrumentos selecionados a priori a partir de hipóteses prévias. Em geral, são comparados indivíduos em condições patológicas específicas e indivíduos não expostos ao fator de risco para alterações cerebrais (controles) com a finalidade de investigar a existência de disfunções cognitivas associadas à patologia em questão. Fatores como idade, escolaridade, uso de medicamentos, uso e abuso de substâncias, entre outros, devem ser controlados a fim de garantir a validade dos resultados encontrados. Além destes, é necessário garantir a confiabilidade entre avaliadores (no caso de mais de um avaliador), a ordem de aplicação dos testes e o efeito de aprendizagem, o que pode ser contornado utilizando-se diferentes formas do mesmo instrumento. 
Por meio de medidas comportamentais, os testes padronizados auxiliam o pesquisador na investigação do funcionamento neuropsicológico, que pode ser afetado por trauma crânio encefálico, distúrbios vasculares, metabólicos ou endócrinos, doenças degenerativas, processos infecciosos, neoplasias, privação de oxigênio e exposição a agentes tóxicos (Lezak, 1995).

A seguir apresentaremos um exemplo de aplicação do método neuropsicológico na pesquisa experimental através de um estudo cujo objetivo consistiu na investigação dos efeitos cognitivos da exposição ao vapor de mercúrio anos após a cessada a exposição.

\section{Avaliação neuropsicológica de indivíduos intoxicados por vapor de mercúrio}

O mercúrio é um metal tóxico, de cor prata e se apresenta em estado líquido na temperatura ambiente. É encontrado na natureza em 3 formas distintas: metálica ou elementar $\left(\mathrm{Hg}^{0}\right)$, formas iônicas ou inorgânicas, que correspondem aos sais de mercúrio derivados dos íons mercuroso e mercúrico $\left(\mathrm{Hg}^{+1}\right.$ e $\mathrm{Hg}^{+2}$, respectivamente) e os compostos orgânicos, resultantes da ação de microrganismos aquáticos sobre as modalidades metálica ou inorgânica (Satoh, 2000).

Atualmente, sob formas elementar e inorgânica, o mercúrio apresenta ampla aplicação no setor industrial, como na metalurgia, petroquímica, produção de lâmpadas, cloro-álcalis, papel, amálgamas dentários, chapéus de feltro e equipamentos médicos, entre outros. O mercúrio elementar também é utilizado nas áreas de garimpo para extração de ouro, atividade que envolve alta concentração do metal no ambiente onde é realizada (Faria, 2003).

O caráter tóxico do mercúrio se deve às várias formas químicas próprias e à alta volatilidade e solubilidade em água e lipídeos, o que facilita a passagem pelos alvéolos pulmonares, pela barreira hemato-encefálica e placenta (Faria, 2003). O sistema nervoso central é considerado o principal órgão afetado na intoxicação crônica pelo vapor de mercúrio elementar em humanos (Agency for Toxic Substance and Disease Registry, 1992). Além disso, a acumulação do mercúrio na célula nervosa parece persistir por toda a vida do individuo (Faria, 2003). O termo eretismo é utilizado para designar as altera- 
ções de comportamento, humor e personalidade como irritabilidade, depressão, timidez, ansiedade, dificuldade de concentração e tomada de decisões, insônia, embotamento intelectual, perda de memória e, nos casos mais graves, apatia, alucinações e delírios, ideação suicida, psicose maníaco-depressiva e alterações de fala (Azevedo, 2003). A exposição ocupacional crônica ao vapor de mercúrio durante período superior a um ano tem sido associada à manifestação de sintomas neurológicos e neuropsicológicos, porém, os estudos são inconclusivos em relação às funções mentais afetadas (Hua, Huang, \& Yang, 1995; Ellingsen, Bast-Pettersen, Efskind, \& Thomassen, 2001; Meyer-Baron, Schaeper, \& Seeber, 2002).

Pesquisas referentes às alterações do sistema nervoso central relacionadas à intoxicação por vapor de mercúrio podem ser divididas entre aquelas cuja avaliação é realizada durante o período de exposição ou após a cessação. Achados de estudos observacionais com trabalhadores industriais (produção de lâmpadas fluorescentes, termômetros ou cloro-álcalis) comparados com controles mostraram déficit de memória operativa (Smith, Langolf, \& Goldberg, 1983), memória verbal de curto prazo (Soleo, Urbano, Petrera, \& Ambrosi, 1990), atenção concentrada, habilidade para cálculos (Liang, Sunn, Chen, \& Li,1993), lentidão psicomotora (Lucchini et al., 2003, Piikivi, Hanninen, Martelin, \& Mantere, 1984), tempo de reação diminuído e incoordenação motora (Lucchini et al., 2003). Foram encontradas correlações entre índice de exposição ao vapor de mercúrio (concentração urinária do metal) e déficits de atenção e memória visual de curto prazo (Ellingsen et al., 2001), funções executivas e memória (Piikivi et al., 1984), além de lentidão psicomotora (Ellingsen et al., 2001).

Pesquisas referentes às alterações neuropsicológicas associadas ao vapor de mercúrio com cessação da exposição anterior à avaliação são escassas, porém, importantes por fornecer informações relevantes quanto à possibilidade de seqüelas ocasionadas pelo contato com o metal. Em estudo cujos participantes eram ex-operários de fábricas de cloro-alcalis foram descritos déficits atencionais e lentidão psicomotora (Mathiesen, Ellingsen, \& Kjuus, 1999). Em outro estudo, ex-trabalhadores de fábricas de cloro-alcalis apresentaram dificuldades significativas nos testes de tempo de reação, destreza manual e memória verbal (Frumkin et al., 1988). Ex-mineradores submetidos previamente a altas concentrações de mercúrio no ar apresentaram desempenho sig- 
nificativamente inferior aos controles nos testes de habilidade sensório-motora, tempo de reação, coordenação motora e viso-motora, destreza manual, controle inibitório, habilidade viso-espacial e memória de curto prazo 18 anos em média após a cessação da exposição. O tempo de afastamento correlacionou-se com o desempenho nos testes de tempo de reação e memória de curto prazo (Kishi et al., 1994).

Este estudo teve como objetivo examinar o funcionamento neuropsicológico de ex-trabalhadores de fábricas de lâmpadas fluorescentes, anos após a interrupção da exposição ocupacional ao vapor de mercúrio metálico. Nossa hipótese era de que estes indivíduos apresentariam um desempenho pior do que os controles nos testes do protocolo.

Foram submetidos à bateria de avaliação neuropsicológica 26 ex-trabalhadores (20 homens e 6 mulheres) de fábricas de lâmpadas fluorescentes, encaminhados pelo Departamento de Medicina Legal, Ética Médica e Medicina Social e do Trabalho da Faculdade de Medicina da Universidade de São Paulo e 20 indivíduos (18 homens e 2 mulheres) sem histórico de exposição crônica a agentes tóxicos, recrutados dentre os funcionários da USP, que constituíram o grupo controle. Dados referentes à concentração de mercúrio urinário dos ex-trabalhadores foram coletados nos prontuários médicos do Hospital das Clinicas da Universidade de São Paulo.

Foram utilizados como critério de exclusão histórico de distúrbios endócrinos ou metabólicos, lesão ou cirurgia cerebral, patologias vasculares, abuso de álcool ou outras drogas e tratamento prévio com agentes quelantes no grupo exposto. A média de tempo de aplicação do protocolo foi de aproximadamente uma hora e 30 minutos.

O estudo foi aprovado pelo Comitê de ética em pesquisa da Universidade de São Paulo e os voluntários foram informados sobre os objetivos e procedimentos da pesquisa e assinaram termo de consentimento informado.

A Tabela 1 mostra os dados demográficos (sexo, idade, escolaridade e trabalho dos participantes. Os ex-trabalhadores expostos ao mercúrio têm idade média de 41,5 \pm 6,1 anos; 9,8 \pm 1,8 anos de educação formal e encontramse afastados do trabalho devido a problemas de saúde decorrentes da exposição ao metal e/ou do trabalho repetitivo na linha de produção de lâmpadas. 
Tabela 1. Características gerais dos participantes expostos ao mercúrio e grupo controle.

\begin{tabular}{|c|c|c|c|c|c|c|c|c|}
\hline & \multicolumn{4}{|c|}{ Grupo Exposto } & \multicolumn{4}{|c|}{ Grupo Controle } \\
\hline & $\mathbf{n}$ & $\mathbf{M} \pm \mathbf{D P}$ & Md & $\%$ & $\mathbf{n}$ & $\mathbf{M} \pm \mathbf{D P}$ & Md & $\%$ \\
\hline Sexo & 26 & & & 100 & 20 & & & 100 \\
\hline Masculino & 20 & & & 77 & 18 & & & 90 \\
\hline Feminino & 6 & & & 23 & 2 & & & 10 \\
\hline Idade (anos) & & $41,5 \pm 6,1$ & 41,5 & & & $42,7 \pm 8,2$ & 42 & \\
\hline Escolaridade (anos) & & $9,8 \pm 1,8$ & 10 & & & $9,7 \pm 2,2$ & 11 & \\
\hline \multicolumn{9}{|l|}{ Trabalho } \\
\hline Empregados & 0 & & & 0 & 20 & & & 100 \\
\hline $\begin{array}{l}\text { Afastados do trabalho devido à } \\
\text { intoxicação por } \mathrm{Hg}^{0} / \text { desempregados }\end{array}$ & 26 & & & 100 & 0 & & & 0 \\
\hline
\end{tabular}

n, número de participantes; $\mathrm{M}$, média; $\mathrm{DP}$, desvio padrão; $\mathrm{Md}$, mediana

Tabela 2. Variáveis específicas do grupo exposto ao vapor de $\mathrm{Hg}^{0}$

\begin{tabular}{|c|c|c|c|}
\hline & $\mathbf{N}$ & $\%$ & $\mathbf{M} \pm \mathbf{D P}$ \\
\hline Tempo de exposição ao vapor de $\mathrm{Hg}^{0}$ (anos) & & & $10,2 \pm 3,8$ \\
\hline Tempo de afastamento do vapor de $\mathrm{Hg}^{0}$ (anos) & & & $6 \pm 4,7$ \\
\hline Concentração urinária de $\mathrm{Hg}^{0}$ durante a exposição $(\mu \mathrm{g} / \mathrm{g} \text { de } \mathrm{Cr})^{*}$ & & & $46.8 \pm 22.1$ \\
\hline Concentração urinária de $\mathrm{Hg}^{0} 1$ ano após cessada a exposição ( $\mu \mathrm{g} / \mathrm{g}$ de $\mathrm{Cr}$ ) & & & $1,8 \pm 0,9$ \\
\hline \multicolumn{4}{|l|}{ Tendinite } \\
\hline Sim & 13 & 50 & \\
\hline Não & 13 & 50 & \\
\hline \multicolumn{4}{|l|}{ Tratamento Medicamentoso } \\
\hline Antidepressivo & 5 & 19 & \\
\hline Ansiolítico & 5 & 19 & \\
\hline Combinados (antidepressivo e ansiolítico) & 6 & 23 & \\
\hline Nenhum & 10 & 39 & \\
\hline
\end{tabular}

Dados de 18 ex-trabalhadores

n, número de participantes; M, média; DP, desvio padrão

A Tabela 2 mostra características clínicas (diagnóstico de tendinite devido ao trabalho repetitivo na linha de produção e uso de medicação psicotrópica) e relacionadas ao contato com vapor de mercúrio do grupo exposto. Conforme dados colhidos nos prontuários desses pacientes, as concentrações urinárias de mercúrio medidas 1 ano após o afastamento da sua função já se encontravam dentro dos limites normais, com média de 1,8 $\pm 0,9 \mu \mathrm{g} / \mathrm{g} \mathrm{Cr}$. 
Foi utilizada uma bateria de testes para avaliação neuropsicológica compreendendo: subteste Dígitos e Reprodução Visual, Wechsler Memory Scale Revised (Wechsler, 1987), subtestes Cubos e Vocabulário, Wechsler Adult Inteligence Scale (Wechsler, 1981), Stroop Test (Spreen \& Strauss, 1998), Buschke Selective Reminding Test (Spreen \& Strauss, 1998), Grooved Pegboard (Lafayette Instrument Corporation, 1979), Controlled Oral Word Association (Spreen \& Strauss, 1998), Wisconsin Card Sorting Test (Heaton, Chelune, Talley, Kay, \& Curtis, 1993). Para a avaliação de sintomas de depressão e ansiedade foram utilizados o Inventário de Ansiedade Traço-Estado (IDATE) (Biaggio \& Natalício, 1979) e o Inventário Beck de Depressão (Cunha, 2001).

Para análise estatística dos resultados foi utilizado o modelo linear geral através de análises conduzidas para cada variável resposta. Transformações logarítmicas foram realizadas para variáveis cuja distribuição não segue o modelo normal. Foram considerados fatores de confusão a idade, o escore no subteste vocabulário, o BDI e a IDATE. Entretanto, como os escores de depressão e ansiedade apresentaram forte correlação entre si, os resultados do IDATE foram retirados do modelo para evitar dados redundantes e foram mantidos apenas os resultados do BDI. Foram incluídos na análise o uso de antidepressivos e ansiolíticos e diagnóstico de tendinite, utilizado somente para análise dos testes que requerem habilidade manual. Para a verificação da associação entre escores obtidos pelos ex-trabalhadores e variáveis relacionadas ao mercúrio (tempo de exposição e de afastamento ao vapor do metal e concentração urinária), utilizou-se testes de correlação de Pearson. As análises foram realizadas no programa estatístico Minitab 14.0. e considerou-se nível de significância de $5 \%$.

O grupo exposto apresentou desempenho significantemente inferior ao grupo controle $(\mathrm{p}<0,05)$ no teste de memória verbal (Selective Reminding Test), controle inibitório (Stroop Test) e destreza viso-motora (Grooved Pegboard). Os ex-trabalhadores mostraram maior lentidão no Stroop Test nas pranchas $1(\mathrm{p}=0,03)$ e $2(\mathrm{p}=0,01)$, mas não na prancha $3(\mathrm{p}=0,09)$, o que sugere lentidão do processamento de informações. Os índices do Selective Reminding Test (memória verbal) que alcançaram significância estatística foram a recuperação de longo prazo $(\mathrm{p}=0,03)$, armazenamento de longo prazo $(\mathrm{p}=0,00)$, re- 
cuperação consistente de longo prazo $(\mathrm{p}=0,01)$ e recuperação tardia $(\mathrm{p}=0,00)$, sugerindo déficits consistentes no processo de evocação verbal espontânea tardia e fixação preservada, uma vez que o índice de reconhecimento não alcançou significância estatística $(\mathrm{p}=0,10)$. O resultado no teste Grooved Pegboard tanto para mão dominante $(\mathrm{p}=0,00)$, como para mão não-dominante $(\mathrm{p}=0,00)$ evidencia lentidão psicomotora no grupo de expostos ao vapor de mercúrio mesmo anos após a cessada a exposição.

Os ex-trabalhadores também obtiveram escores significantemente maiores nos inventários BDI e IDATE, indicando sintomatologia depressiva e de ansiedade ( $\mathrm{p}<0,001$ para todos os casos; dados não tabulados). A concentração de mercúrio urinária medida 1 ano após a cessação da exposição correlacionou-se com escores do IDATE apenas para o traço de ansiedade ( $\mathrm{r}=0,75$; $\mathrm{p}=0,03$; Correlação de Pearson). O nível de mercúrio durante a exposição e os tempos de exposição e de afastamento do mercúrio não apresentaram correlações significativas com os escores neuropsicológicos.

$\mathrm{Na}$ análise das variáveis específicas do grupo de expostos, o aumento da concentração urinária de mercúrio acompanhou a progressão dos escores de traço de ansiedade (IDATE). Dados prévios da literatura mostram a presença de sintomas subjetivos relacionados a alterações de humor e personalidade mediante concentrações baixas de mercúrio (Langworth, Almkvist, Söderman, \& Wikström, 1992). Mulheres com obturações odontológicas de amálgama de mercúrio obtiveram escores significantemente superiores no IDATE em comparação àquelas sem restaurações nas questões referentes à autoconfiança (Siblerud, Motl, \& Kienholz, 1994). Por outro lado, o tempo de exposição ao vapor de mercúrio e de afastamento da fonte de exposição não se correlacionou com nenhum dos testes ou inventários aplicados. Entretanto, vale ressaltar que os índices de exposição são valores imprecisos, pois dependem do cargo que cada indivíduo ocupava.

\section{Considerações Finais}

O conhecimento do cérebro e suas funções têm desafiado os cientistas desde a antiguidade, levando-os a construir e reconstruir teorias que possam dar conta de conceitos complexos como comportamento, emoção e cognição. 
A complexidade do conhecimento alcançado pelas diferentes disciplinas que compõem as chamadas Neurociências evidencia cada vez mais a necessidade de troca entre elas, exigindo do pesquisador uma formação cada vez mais abrangente. Neste cenário, a Neuropsicologia se insere desenvolvendo métodos cada vez mais sofisticados, passando a interagir com áreas como ressonância magnética funcional e biologia molecular. É a partir destas infinitas possibilidades que se cria e recria o conhecimento.

Taub, A., Zachi, E. C., Teixeira, R. A. A., \& Ventura, D. F. (2006). Neuropsychology in experimental research: Mercury intoxication as an example of application. Psicologia USP, 17(4), 287-300.

\begin{abstract}
The development of the field of neuropsychology followed the study of the brain since its oldest records. Neuropsychological science uses quantitative measures of performance and technologies for the production of neuroimage that, jointly, allow the observation of the brain-behavior relationship. Neuropsychological research has revealed results in the investigation of cognitive, psychomotor or behavioral alterations related to congenital or acquired pathologies. In the present article we describe historical aspects of experimental neuropsychology and present the main results of a study about neuropsychological alterations found in patients with a history of mercury vapor intoxication, which persisted years after exposure.
\end{abstract}

Idex terms: Neuropsychology. Neuropsychological assessment. Mercury poisoning.

Taub, A., Zachi, E. C., Teixeira, R. A. A., \& Ventura, D. F. (2006). La neuropsychologie en recherche experimental: l'intoxication par mercure comme un exemple d'application. Psicologia USP, 17(4), 287-300. 
Résumé: Le dévelopment de la neuropsychologie a accompagné l'étude du cerveau dès ces plus anciens régistres. La science neuropsychologique s'utilise des mésures quantitatives de performance et des téchnologies pour la production de neuroimage, qui permettent l'observation des relations cerveau-comportement. Sur le champ experimental, la neuropsychologie a revelé des résultats dans les investigations des alterations cognitives, psychomotrices, ou comportamentales associés aux patologies génétiques ou acquises. Dans cet article on abordera les aspects historiques de la neuropsychologie experimental. Aussi on presentera les principaux résultats d'un étude référant aux altérations neuropsychologiques des pacients avec une histoire d'intoxication de vapeur de mercure présent après des années d'exposition au même.

Mots-clés: Neuropsychologie. Evaluation neuropsychologique. Intoxication mercurie.

\section{Referências}

Agency for Toxic Substance and Disease Registry. (1992). Mercury toxicity. Environmental Medicine, 46(6), 1731-1741.

Azevedo, F. A. (2003). Toxicologia do mercúrio. São Paulo: InterTox.

Biaggio, A. M. B., \& Natalício, L. (1979). Manual do Inventário de Ansiedade Traço. Rio de Janeiro: CEPA.

Branco, D., \& da Costa, J. C. (2006). Ressonância magnética funcional de memória: onde estamos e onde podemos chegar. Journal of Epilepsy and Clinical Neurophysiology, 12(1), 25-30.

Cunha, J. A. (2001). Manual da versão em português das Escalas Beck. São Paulo: Casa do Psicólogo.

Ellingsen, D. G., Bast-Pettersen, R., Efskind, J., \& Thomassen, Y. (2001). Neuropsychological effects of low mercury vapor exposure in chloralkali workers. Neurotoxicology, 22(2), 249-258.

Faria, M. A. M. (2003). Mercurialismo metálico crônico ocupacional. Revista de Saúde Pública, 37(1), 116-127.

Feinberg, T. E., \& Farah, M. L. (1997). Behavioral neurology and neuropsychology. New York: McGraw-Hill.

Frumkin, H., Letz, R., Williams, P.L., Gerr, F., Pierce, M., Sanders, A. et al. (2001). Health 
A Aplicação da Neuropsicologia na Pesquisa Experimental: o Exemplo...

effects of long-term mercury exposure among chloralkali plant workers. American Journal of Industrial Medicine, 39, 1-18.

Gil, R. (2002). Neuropsicologia hoje (2a ed.). São Paulo: Santos.

Heaton, R. K., Chelune, G. J., Talley, J. L., Kay, G. G., \& Curtis, G. (1993). Wisconsin Card Sorting Test: Manual revised and expanded. Odessa, TX: Psychological Assessment Resources.

Hua, M. S., Huang, C. C., \& Yang, Y. J. (1995). Chronic elemental mercury intoxication: Neuropsychological follow-up case study. Brain Injury, 10, 377-384.

Kishi, R., Doi, R., Fukuchi, Y., Satoh, H., Satoh. T., Ono, A. et al. (1994). Residual neurobehavioral effects associated with chronic exposure to mercury vapour. Occupational and Environmental Medicine, 51, 35-41.

Lafayette Instrument Corporation USA. (1979). Grooved pegboard instruction: Owner's manual. Indiana: Author.

Langworth, S. Almkvist, O., Söderman, E., \& Wikström, B. O. (1992). Effects of occupational exposure to mercury vapour on the central nervous system. British Journal of Industrial Medicine, 49, 545-55.

Lezak, M. D. (1995). Neuropsychological assessment (2nd ed.). New York: Oxford.

Liang, Y., Sunn, R., Chen, Z., \& Li, L. (1993). Psychological effects of low exposure to mercury vapor: Application of a computer-administered neurobehavioral evaluation system. Environmental Research, 60, 320-327.

Lucchini, R., Calza, S., Camerino, D., Carta, P., Decarli, A., Parrinelo, G. et al. (2003). Application of a latent variable model for a multicenter study on early effects due to mercury exposure. NeuroToxicology, 24, 605-616.

Luria, A. R. (1981). Fundamentos de neuropsicologia. São Paulo: Edusp.

Magila, M. C., \& Xavier, G. F. (2000). Interação entre sistemas e processos de memória em humanos. Temas em Psicologia, 8(2), 143-154.

Mathiesen, T., Ellingsen, D. G., \& Kjuus, H. (1999). Neuropsychological effects associated with exposure to mercury vapor among former chloralkali workers. Scandinavian Journal of Work, Environment and Health, 25(4), 342-350.

Meneses, M. S., Rocha, S. F. B., Blood, M. R. Y., Trentin, A., Benites Filho, B. R., Kovacs, P. A. et al. (2004). Ressonância magnética funcional na determinação da lateralização da área cerebral da linguagem. Arquivos de Neuro-Psiquiatria, 62(1), 61-67.

Meyer-Baron, M., Schaeper, M., \& Seeber, A. (2002). A meta-analysis for neurobehavioral results due to occupational mercury exposure. Archives of Toxicology, 76, 127-136. 
Moll, J., de Oliveira-Souza, R., Eslinger, P. J., Bramati, I. E., Mourao-Miranda, J., Andreiuolo, P. A. et al. (2002). The neural correlates of moral sensitivity: A functional magnetic resonance imaging investigation of basic and moral emotions. The Journal of Neuroscience, 22(7), 2730-2736.

Piikivi, L., Hanninen, H., Martelin, T., \& Mantere, P. (1984). Psychological performance and long-term exposure to mercury vapors. Scandinavian Journal of Work, Environment and Health, 10(1), 35-41.

Satoh, H. (2000). Occupational and environmental toxicology of mercury and its compounds. Industrial Health, 38, 153-164.

Siblerud, R. L., Motl, J., \& Kienholz, E. (1994). Psychometric evidence that mercury from silver dental fillings may be an etiological factor in depression, excessive anger, and anxiety. Psychological Reports, 74, 67-80.

Smith, P. J., Langolf, G. D., \& Goldberg, J. (1983). Effects of occupational exposure to elemental mercury on short-term memory. British Journal of Industrial Medicine, 40, 413-419.

Soleo, L., Urbano, M. L., Petrera, V., \& Ambrosi, L. (1990). Effects of low exposure to inorganic mercury on psychological performance. British Journal of Industrial Medicine, 47(2), 105-109.

Spreen, O., \& Strauss, E. (1998). A compendium of neuropsychological tests. New York: Oxford.

Volchan, E., Pereira, M. G., Oliveira, L., Vargas, C., Mourão-Miranda, J., Azevedo, T. M. et al. (2003). Estímulos emocionais: processamento sensorial e respostas motoras. Revista Brasileira de Psiquiatria, 25(Supl. 2), 29-32.

Wechsler, D. (1981). Wechsler adult inteligence scale revised manual. New York: Psychological Corporation.

Wechsler D. (1987). Wechsler memory scale-revised manual. San Antonio, TX: The Psychological Corporation.

Recebido em: 31/10/2006 Aceito em: 01/12/2006 